\title{
Functional connectivity studies of patients with auditory verbal hallucinations
}

\author{
Ralph E. Hoffman ${ }^{1 *}$ and Michelle Hampson ${ }^{2}$ \\ ${ }^{1}$ Department of Psychiatry, Yale University School of Medicine, New Haven, CT, USA \\ 2 Department of Diagnostic Radiology, Yale University School of Medicine, New Haven, CT, USA
}

\section{Edited by: \\ Kenneth Hugdahl, University of \\ Bergen, Norway}

Reviewed by:

Kenneth Hugdahl, University of Bergen, Norway

Claude Alain, Rotman Research

Institute, Canada

\section{*Correspondence:}

Ralph E. Hoffman, Yale-New Haven Psychiatric Hospital, 184 Liberty Street LV108, New Haven, CT 06519, USA.

e-mail: ralph.hoffman@yale.edu
Functional connectivity (FC) studies of brain mechanisms leading to auditory verbal hallucinations (AVHs) utilizing functional magnetic resonance imaging (fMRI) data are reviewed. Initial FC studies utilized fMRI data collected during performance of various tasks, which suggested frontotemporal disconnection and/or source-monitoring disturbances. Later FC studies have utilized resting (no-task) fMRI data. These studies have produced a mixed picture of disconnection and hyperconnectivity involving different pathways associated with AVHs. Results of our most recent FC study of AVHs are reviewed in detail. This study suggests that the core mechanism producing AVHs involves not a single pathway, but a more complex functional loop. Components of this loop include Wernicke's area and its right homologue, the left inferior frontal cortex, and the putamen. It is noteworthy that the putamen appears to play a critical role in the generation of spontaneous language, and in determining whether auditory stimuli are registered consciously as percepts. Excessive functional coordination linking this region with the Wernicke's seed region in patients with schizophrenia could, therefore, generate an overabundance of potentially conscious language representations. In our model, intact FC in the other two legs of corticostriatal loop (Wernicke's with left IFG, and left IFG with putamen) appeared to allow hyperconnectivity linking the putamen and Wernicke's area (common to schizophrenia overall) to be expressed as conscious hallucinations of speech. Recommendations for future studies are discussed, including inclusion of multiple methodologies applied to the same subjects in order to compare and contrast different mechanistic hypotheses, utilizing EEG to better parse time-course of neural synchronization leading to $\mathrm{AVH}$ s, and ascertaining experiential subtypes of $\mathrm{AVH}$ s that may reflect distinct mechanisms.

Keywords: auditory verbal hallucinations, schizophrenia, functional connectivity, functional magnetic resonance imaging, Wernicke's area, putamen, speech
Between $60 \%$ and $80 \%$ of patients with schizophrenia experience auditory verbal hallucinations (AVHs) of spoken speech (Sartorius et al., 1974; Andreasen and Flaum, 1991). These hallucinations often produce high levels of distress and functional disability and are resistant to conventional treatments. Understanding the mechanism of AVHs may shed light on the pathophysiology of schizophrenia overall, and lead to more specific treatments. Mechanistic models under consideration include abnormalities involving source attribution of ordinary inner speech, abnormalities involving registration and retrieval of acoustic memories, and bottom-up activation of speech processing neurocircuitry (for review see Jones, 2010).

Besides studies of activation associated with AVH occurrences, functional magnetic resonance imaging (fMRI) offers another tool for probing the mechanism of AVHs, namely functional connectivity (FC). FC refers to computational measures derived from inter-region correlations of blood-oxygen-level dependent (BOLD) activity time-course. In theory, FC reflects either direct cross-region interactions or correlated activity arising from inputs from a third, shared source. Computational strategies have been developed that minimize effects of non-neural factors such as vascular physiology and respiration, using, for example, lowpass filtering signals (generally employing a $0.1 \mathrm{~Hz}$ cut-off) and removing variance corresponding to slice or whole brain mean time-course. Resting or "no-task" FC has been able to identify functional pathways critical to normal vision, motor function, audition, language, reading, and attention (Biswal et al., 1995; Lowe et al., 1998; Xiong et al., 1999; Cordes et al., 2000; Hampson et al., 2002, 2006; Fox et al., 2006; Shmuel and Leopold, 2008). These advances raise the possibility that FC mapping can reveal neural pathway abnormalities producing AVHs.

\section{FC STUDIES OF AVHs USING FMRI DATA COLLECTED DURING TASK PERFORMANCE}

Initial studies of AVH mechanisms utilizing FC incorporated different behavioral tasks during fMRI data acquisition. The first such study was reported by Lawrie et al. (2002), who compared eight patients with schizophrenia and 10 healthy controls using fMRI data collected during a sentence completion task. Correlations of BOLD activity time-course in the left 
DLPC and left middle/superior temporal regions were reduced in patients with schizophrenia when compared to data from controls ( $t=1.8, d f=14, p<0.051$-tailed). Within the patient group, $3 / 8$ patients were hallucinators. These patients had lower correlation scores relative to the non-hallucinating patients $(p<0.05)$. The authors hypothesized that these results reflected frontotemporal pathway disruptions that bring about AVHs. Along these lines, a leading hypothesis is that AVHs are instances of inner speech misidentified as non-self-due to a disruption of frontotemporal efference copy projections that ordinarily signal to sensory systems that actions (and thought) are self-generated (Feinberg, 1978; Frith and Done, 1988; Ford et al., 2007; HeinksMaldonado et al., 2007; Jones, 2010).

In a second study, Shergill et al. (2003) studied eight hallucinating patients with schizophrenia and eight healthy control subjects. Two conditions were compared, self-paced inner speech generated once per second (repeating a single word, "rest") or the same word repeated once every 4 seconds. Relative to the control group, the patient group exhibited reduced correlation between left inferior frontal activity and activity in the right middle and superior temporal gyri, the right insula and a region encompassing the right parahippocampal, inferior temporal, and fusiform gyri. These findings were interpreted as indicating a source monitoring impairment with reduced information flow from frontal areas to posterior regions.

Using a different behavioral task involving perception of external speech, Mechelli et al. (2007) studied 10 hallucinating patients with schizophrenia, 10 non-hallucinating patients with schizophrenia and 10 healthy controls with fMRI. Stimuli consisted of pre-recorded single-word utterances. Some were recordings of words previously spoken by the subject, others were words generated by the subject during the same recording sessions but recorded by someone else, and a third set of stimuli were recorded by the subject, but pitch-shifted -0.4 semitones. Using dynamic causal monitoring applied to fMRI data, the functional impact of one region on another (referred to as "effective connectivity") was assessed relative to these three stimulus conditions. A total of five a priori cortical regions were definedanterior cingulate, left inferior frontal, right inferior frontal, left posterior superior temporal, and right posterior temporal. This yielded a total of 20 ordered linkages that were analyzed for group differences. Using a corrected statistical threshold of 0.01 , the impact of left superior temporal on anterior cingulate activity was found to be greater for non-self-spoken words compared to self-spoken words for healthy controls and nonhallucinating patients; however, this pattern was reversed for hallucinators, i.e., self-spoken speech produce greater effective connectivity along this pathway compared to non-self-spoken speech. These data, therefore, suggest some source monitoring alteration exhibited by hallucinators. That the direction of causal mapping for the critical pathway is posterior-to-frontal is not surprising given that: (1) this was a perceptual task; (2) non-self-produced speech requires auditory perceptual processing that is probably greater in scope than self-spoken words due to source identification processes. That this differential condition effect is reversed in hallucinators is of considerable interest. It is possible that somehow perceptual or source monitoring indicators of self vs. non-self-speech during perceptual processing are somehow switched. How this might happen remains uncertain.

\section{RESTING FC STUDIES OF AVH PATHOPHYSIOLOGY}

Resting or no-task FC fMRI studies have been used more recently to study AVH pathophysiology. These approaches offer the possibility of detecting spontaneous or baseline network interactions as predisposing factors leading to AVHs since no specific task is utilized during data collection. Four such FC studies of AVHs in patients with schizophrenia are outlined below:

Vercammen et al. (2010a) compared a relatively large sample of patients with active AVHs $(N=27)$ with healthy controls $(N=27)$ using resting state FC. FC was calculated for seed regions reflecting the left and right temporoparietal junction (TPJ) based on a previous study suggesting that both these cortical regions play an important role in the genesis of AVHs (Hoffman et al., 2007). Their patient group demonstrated subnormal left TPJ FC linking with the right homotope of Broca's area. There was no non-hallucinating patient group with schizophrenia in this study for comparison. However, within the group of hallucinators, more severe AVHs were associated with reduced FC linking the left TPJ seed region and the bilateral anterior cingulate and bilateral amygdala.

In a follow-up study, Vercammen et al. (2010b) rescanned 18 patients with schizophrenia after a six-day course of $1 \mathrm{~Hz}$ repetitive transcranial magnetic stimulation (rTMS) to the left TPJ or sham stimulation to the same location. FC relative to TPJ seed regions was assessed. Some studies have demonstrated that intervention has efficacy in reducing AVHs (Hoffman et al., 2005; Poulet et al., 2005). Although no corresponding changes were observed in FC following rTMS that at baseline were statistically associated with AVH severity, an increase in FC between the left TPJ and the right insula was observed in the group receiving active rTMS. This result is of interest given a number of studies finding activation in the insula associated with AVH events (for meta-analysis of activation studies of AVHs see Jardri et al., 2011). Moreover, these data suggest that low-frequency rTMS can enhance FC linking some regions.

A second study of AVHs utilizing resting FC was subsequently reported by Gavrilescu et al. (2010). Their group examined crosshemisphere FC linking the primary and secondary auditory cortices in 13 patients with schizophrenia and AVHs, 13 similarly diagnosed patients without AVHs, and 16 healthy controls. Their selection of pathway was based on a prior neuropsychological study of AVHs suggesting abnormal transcallosal transfer of information in this patient group (Green et al., 1994). In the Gavrilescu study, separate FC assessments were computed for primary and secondary auditory cortex using regions of interest (ROI) determined from fMRI data collected while patients listened to words. This is a notable strength of the study insofar as this approach adjusts for the fact that localization of language functions is quite variable across subjects (cf. Ojemann, 1991). FC was estimated as the cross-correlation of primary and secondary auditory BOLD activity. Hallucinators demonstrated significant reductions in inter-hemispheric FC compared to the other two groups. As noted 
by the authors, one limitation of their study is that this approach is not able to differentiate whether transcallosal disconnection was due to occurrence of hallucinations themselves or whether this finding reflected a vulnerability factor that is relatively sustained.

Raij et al. (2009) reported a third study of AVHs utilizing a measure related to FC based on at rest fMRI data. There was no comparison between hallucinating and non-hallucinating patient subgroups; instead shifts in coupling elicited by the on-off occurrences of hallucination events themselves were determined. These occurrences were signaled by patients during scanning. A total of 11 subjects who actively hallucinated during scanning were studied. Seed regions were defined in the left and right IFG. Specific ratings of level of reality and loudness of AVHs on an event-by-event basis were generated by each patient during scanning. Patient ratings of fluctuating levels of experienced reality of AVHs were found to correlate positively with hallucinationspecific coupling linking the left IFG with bilateral auditory cortex, the right posterior temporal lobe, the middle right anterior cingulate cortex, the right ventral striatum, and the left nucleus accumbens. A correlation between hallucination reality and right IFG coupling with the right posterior temporal lobe was also detected. These findings, therefore, appeared to support the view that elevated FC along certain pathways makes AVHs worse. This view was further reinforced by our own studies described below.

\section{FC STUDIES OF AVHs BY OUR GROUP}

The first FC examination of AVHs reported by our group was incorporated into a larger study using fMRI methods to position rTMS in an attempt to curtail these experiences in the context of a clinical trial (Hoffman et al., 2007). Our primary finding was that left Wernicke's FC with the right homologue of Broca's area was strongly and negatively correlated with rTMS response to this region (Spearman-rank correlation $=-1.0, p<0.001$ ) in patient group with continuous, non-stop hallucinations. Although based on a small number of subjects $(N=6)$, this correlation suggests that IFG/STG linkages reinforce pathophysiology, thereby rendering AVHs less reversible by rTMS.

Our group subsequently reported the largest FC study of AVHs to date, comparing 32 patients with schizophrenia-spectrum disorder and AVHs with similarly diagnosed patients without AVHs $(N=24)$ and healthy controls $(N=23$; Hoffman et al., 2011). Non-hallucinating patients either never experienced AVHs, or if they did not within the five years prior to scanning. For the purposes of this study, FC was seeded from a bilateral Wernicke's region delineated according to BOLD activation detected when contrasting hallucination periods with non-hallucination periods that were signaled by a prior non-overlapping group of patients during fMRI scanning (Hoffman et al., 2008). This seed region was located in the left posterior superior temporal gyrus (STG) combined with a roughly homologous region in the right posterior STG and the neighboring middle temporal gyrus (MTG). This approach had not been previously employed in an FC study of AVHs, and potentially optimizes chances of accessing directly neurocircuitry involved in the genesis of these experiences. FC relative to this seed region was computed using methods involving low-pass filtering (frequency cutoff $0.1 \mathrm{~Hz}$ ) and statistically removing effects of the average time-course of the slice in which the pixel was located, consistent with previous resting connectivity analyses (Hampson et al., 2002, 2006).

Unpublished pilot data based on another sample of subjects prompted us to predict that FC between the Wernicke's seed region and subregions of the left IFG would be elevated in the hallucinator group relative the non-hallucinator group and healthy controls. This prediction was assessed using a region-of-interest analysis. In addition, we undertook an exploratory voxel-based analysis to search for other sites showing group FC differences.

As predicted, Wernicke's-seeded FC with Brodmann Area 45/46 in the left IFG was significantly greater for hallucinating patients compared to non-hallucinating patients, but, contrary to our pilot data, not compared to healthy controls. The exploratory, voxel-based analysis did not reveal differences in any other brain region for bilateral Wernicke's-seeded FC comparing hallucinating and non-hallucinating patients after FDR correction (cut-off 0.05). However, a large cluster of subcortical voxels exhibited excessive FC relative to the Wernicke's seed region when comparing both patient groups with healthy controls following FDR correction. This cluster incorporated major components of the thalamus, midbrain, and putamen bilaterally.

In order to further probe the mechanism of AVHs, a seed region was defined in left IFG using a cut-off of 0.005 contrasting hallucinating and non-hallucinating patients. We furthermore divided the subcortical cluster described above into three ROIs, and calculated FC in a standard fashion, now relative to the left IFG seed region. One of these regions, the putamen, demonstrated excessive IFG-seeded FC when hallucinators were contrasted with non-hallucinating patients $(t(52)=$ 2.7, $p=0.009 \times 3=0.027$ after Bonferroni correction). IFGseeded FC with the putamen, Wernicke's-seeded FC with the putamen, and Wernicke's-seeded FC with the IFG seed region were consequently summed together as a corticostriatal loop. This composite FC measure demonstrated a significant group effect $(F(2,76)=9.84, p=0.0002)$, with hallucinators greater than both non-hallucinating patients and healthy controls, who were not different from each other (Duncan pairwise comparisons with $\alpha=0.05)$. Pooling data from the two patient groups, correlations between corticostriatal FC effects of antipsychotic drug dose (tallied as chlorpromazine equivalents) was assessed and found to be non-significant $(R=0.08)$. To determine if chronicity of illness contributed to our findings, correlation between network FC and number of hospitalizations was computed and found also to be non-significant $(R=-0.21)$.

Relevant to the Vercammen et al. (2010a) findings, we found that FC was reduced between the bilateral Wernicke's seed region and the anterior cingulate in patients compared to controls; however, this finding did not differentiate hallucinating and nonhallucinating patient subgroups. Along similar lines, we found that FC between the bilateral Wernicke's seed region and a subregion in left IFG (BA47) to be reduced in patients. This finding is consistent with other studies demonstrating partial frontotemporal disconnection in hallucinators (Lawrie et al., 2002; Shergill et al., 2003). However, these FC data in our study 
also did not differentiate hallucinating and non-hallucinating patients. Therefore, our FC data, based on a larger sample of hallucinating patients with well-matched comparison groups of non-hallucinating patients and healthy controls, suggest overall that these corticocortical pathways are unlikely to play a primary mechanistic role in the generation of AVHs.

We found instead that the only pathway demonstrating hyperconnectivity differentiating hallucinators and healthy controlsat least in terms of the seed regions selected-was between the putamen and the bilateral Wernicke's seed region. However, in terms of specificity of findings, a parallel group difference was also detected between non-hallucinating patients with schizophrenia. How can one make sense of these FC findings, considered as whole?

One clue is that the putamen appears to play a critical role in initiating language representations (Price, 2010). Moreover, FC linking the putamen to diverse cortical regions plays a critical role in determine whether external auditory stimuli are experienced as conscious percepts (Mhuircheartaigh et al., 2010) Excessive functional coordination linking this region with the Wernicke's seed region in patients with schizophrenia could, therefore, generate an overabundance of potentially conscious language representations. Intact FC in the other two legs of corticostriatal loop (Wernicke's $\leftrightarrow$ left IFG, and left IFG $\leftrightarrow$ putamen) appeared to allow this disturbance (common to schizophrenia overall) to be expressed as a conscious hallucination of speech. Disruption in one or both of these linkages, in essence, appears to protect patients from experiencing overabundant, spontaneous language representations as (spurious) externalized percepts of spoken speech. It is generally assumed that disconnection leads to greater functional disturbance. However, our data support a more complex view whereby hyperconnectivity intrinsic to one component in a functional loop may be counterbalanced by hypoconnectivity in other components of that loop.

These study results are consistent with a recent study demonstrating that treatment with second-generation antipsychotic medications in patients with schizophrenia produces widespread reductions in FC that may be related to their therapeutic effects (Lui et al., 2010). The prediction of this study is that curtailment of connectivity in any of the three legs of the corticostriatal loop (Wernicke's $\leftrightarrow$ left IFG, and left IFG $\leftrightarrow$ putamen or putamen $\leftrightarrow$ Wernicke’s) would produce a reduction in AVHs.

These findings challenges inner speech models of AVHs based on disconnection of monitoring processes or efferent copy signaling (for review see Jones, 2010; see also Heinks-Maldonado et al., 2007) since no disconnected pathway is identified as a primary causal factor. These findings also challenge acoustic memory explanatory models (Jones, 2010) since involvement of medial temporal regions ordinarily involved in these memory processes was not linked with AVH vulnerability. However, these findings are consistent with a speech perception network activity model of AVHs termed "social deafferentation" (Jones, 2010). This model is based on the fact that these patients, prior to onset of psychosis, are generally in a state of social withdrawal (for original description of this model see Hoffman, 2007). Hallucinated speech could then a brain response to "fill in the blank" of relatively absent conversational discourse in the real world given that the human brain is highly dedicated to processing and understanding spoken language ordinarily. The social deafferentation model predicts lowered threshold-to-consciousness of language representations analogous to other types of hallucinations generated from sensory deafferentation states (such as visual deprivation) where there is also lowering of threshold-to-consciousness. Hyperconnectivity linking the putamen and Wernicke's area could be the means whereby lowered threshold-to-consciousness of conversational language representations occurs in patients with schizophrenia.

\section{DISCUSSION}

The FC studies reviewed above have produced divergent results. Reasons for this divergence are likely numerous.

First, the task-related FC studies outlined above each utilized different behavioral tasks and utilized small numbers of subjects, making more general conclusions difficult. It is also hard to integrate findings across the studies reviewed above since, taskbased FC reflects different neural processes compared to no-task FC. This conundrum is suggested by a meta-analysis reported by Kompus et al. (2011), who pooled results fMRI and PET activation studies of spontaneous AVH events in parallel with results of activation studies conducted utilizing external auditory stimuli in patients with these symptoms. A paradox was detected: robust, spontaneous activation was detected in the left primary auditory cortex and other regions concurrent with hallucination events, even though responses to external auditory stimuli were subnormal for hallucinators. The authors suggested that this discrepancy was due either to an attentional bias toward internal events, or some failure of a default network to deactivate when auditory processing neurocircuitry is engaged by external stimuli. An analogous situation may hold for FC ascertained along some pathways: engagement in a listening task (see for instance Mechelli et al., 2007) may elicit subnormal activated FC in hallucinators compared to controls-possibly due to a failure to deactivate default processing, whereas spontaneous FC in hallucinators along similar pathways may still be normal or supranormal.

Another source of study outcomes differences derives from the fact that, when calculating FC, data analytic methods are applied in order to minimize spurious sources of covariance such as breathing, cardiac pulse, and other brain-wide shifts in activation. These methods varied considerably from one study to the next, which will lead to very different results.

One serious limitation is that only three of eight FC studies reviewed above compared hallucinating and non-hallucinating patients (Mechelli et al., 2007; Gavrilescu et al., 2010; Hoffman et al., 2011), which is an important test of any mechanistic model of this symptom.

Two of three no-task FC studies of AVHs have highlighted disconnection as a factor, one considering cross-hemispheric linkages (Gavrilescu et al., 2010), and the other (Vercammen et al., 2010a) considering a linkage similar to that considered by Mechelli et al. (2007). However, implications of the Vercammen et al. findings are uncertain since the FC linkage correlating with hallucination severity was not the FC linkage that was 
abnormal in the patient group compared to healthy controls. The Vercammen et al. study could be interpreted as indicating that reduced left temporoparietal $\leftrightarrow$ right Broca's FC is the primary abnormality, with relative reductions in FC linking the former with bilateral anterior cingulate and the amygdala being permissive in allowing AVH experiences to emerge. Our data did not confirm these FC relationships and group differences. Perhaps the reason for this discrepancy is that our definition of a temporoparietal seed region was different, involving Wernicke's areas proper plus a right homologous pooled site based on a prior fMRI activation study of AVH events (Hoffman et al., 2008). Another uncertainty regarding the Vercammen et al. findings is that the functional capacities of right Broca's area are not well understood. This region appears to have some residual linguistic processing capacity elicited by tasks such as word reading that can be detected with neuroimaging following left IFG lesions (Rosen et al., 2000). How disconnection of this functional capacity relative to left TPJ might increase vulnerability to AVHs is not clear.

In contrast, our corticostriatal FC findings and those reported by Raij et al. (2009) suggest that heightened FC along certain pathways plays an important role in the genesis of AVHs. A hybrid model consequently has been proposed by one of us (Ford and Hoffman, 2012) whereby "locked in" speech perception neurocircuitry dynamics arising from network hyperconnectivity both: (1) lowers threshold-to-consciousness of language representations, and (2) overrides corollary discharge signals from frontal areas to produce a source monitoring defect; in this scenario, both factors contribute to the genesis of hallucinatory experience. A related model has been proposed by Hugdahl (2009), whereby perceptual neurocircuitry is responsible for key aspects of hallucinatory experience, with a failure of top-down processing amplifying a specific (experiential, etc.) dimension, namely the sense that the experience derives from an external source.

It is noteworthy that disconnection along some pathways is not inconsistent with hyperconnectivity along other pathways; in fact the two types of findings may be related. For instance, hyperconnectivity linking regions $\mathrm{A}$ and $\mathrm{B}$ will tend to depress indirectly FC linking regions $\mathrm{B}$ and $\mathrm{C}$. The reason for this is that elevating variance detected in $\mathrm{B}$ referable to $\mathrm{A}$ will tend to mask variance in $\mathrm{B}$ referable to $\mathrm{C}$-thereby depressing FC between the latter two regions. Moreover the opposite is also true: decreasing variance detected in $\mathrm{B}$ referable to A could unmask variance in B shared with $\mathrm{C}$-hereby increasing FC linking the latter two regions.

In terms of future studies, we believe that a numerous refinements in method are indicated to clearly demarcate the mechanism of AVHs.

First, future FC studies need to consider specific loops and networks, not single pathways.

Second, complex data acquisition protocols applied to same subjects should be used to directly compare and contrast competing explanatory models of AVHs. For instance, we are now undertaking a study that examines resting FC and FC during the inner speech task described by Shergill et al. (2003) to ascertain the relationship between corticostriatal loop abnormalities and efference copy disconnection.

Third, it is critically important to more clearly differentiate between findings reflecting sustained vulnerability factors leading to AVHs versus processes occurring simultaneous and downstream of actual occurrences of AVHs. An examination of our corticostriatal FC data, for instance, included consideration of effect of objective rate of hallucinations for those patients $(N=10)$ signaling these events with button-presses during scanning. A promising correlation between corticostriatal loop FC and hallucination frequency was detected, although not statistically significant (Spearman rho $=0.50, p=0.11$ ), possibly due to the small number of subjects who were able to complete this task. Our hypothesis is that there is a dynamic process at play whereby baseline elevations in corticostriatal FC (reflecting a sustained vulnerability factor) lead to further transient increases in FC that occur episodically and correspond to hallucination experiences themselves. Methods capable of more fine-grained analysis of temporal time-course, such as EEG or MEG would be helpful to more fully clarify this issue by looking for synchronization "spikes" simultaneous with occurrence of AVH events.

Fourth, these studies will likely be advanced if different phenomenological types of AVHs are ascertained. Our experience with this population suggests at least three subtypes of AVHs. The first is the "standard" type, where hallucinations are intermittent, spontaneous, with clear breaks between events. This is the dominant form of hallucination among patients with schizophrenia, and they generally respond to currently available treatments, including medication and/or rTMS. However, there are two other hallucination subtypes that we have found to be much more treatment-resistant, suggesting a somewhat different mechanism. The first are patients whose AVHs occur continuously during wakefulness (i.e., no intervening "silent" periods). These patients prompted our first FC study that was combined with rTMS, and produced a somewhat different pathophysiological model highlighting interactions between posterior temporal regions and the right homologue of Broca's area as critical in generating AVHs (Hoffman et al., 2007). The third type of AVHs consists primarily of perceptual transformations of actual spoken speech generated in the environment with some phonetic unclarity, such as mumbled speech in a crowd, rapid speech on the television, or song. These instances of AVHs, which result in spurious, self-referential verbal percepts, seem clearly to arise from aberrant and excessive top-down processing, and are worthy of targeted study in their own right. Other critical dimensions are the overall sense of reality of AVHs (cf. Raij et al., 2009), perhaps best characterized by the degree that patients differentiate these experiences from their own inner speech (Hoffman et al., 2008). A more precise characterization of AVH subtypes may sharpen characterization of corresponding neurocircuitry.

Fifth, it will be important to ascertain whether the connectivity abnormalities delineated by such studies in patients with schizophrenia produce specific cognitive or language processing impairments outside of producing AVHs.

Overall these FC studies have raised important questions regarding the mechanism of AVHs which are addressable by new, improved study designs.

\section{ACKNOWLEDGMENTS}

This work was supported by National Institute of Mental Health Grant R01MH067073, a Dana Foundation grant, a 
National Alliance for Research on Schizophrenia and Depression Independent Investigator Award, Peterson 50th Anniversary Research Partner, and the Department of Mental Health and

\section{REFERENCES}

Andreasen, N. C., and Flaum, M. (1991). Schizophrenia: the characteristic symptoms. Schizophr. Bull. $17,27-50$.

Biswal, B. B., Yetkin, F. Z., Haughton, V. M., and Hyde, J. S. (1995). Functional connectivity in the motor cortex of resting human brain using echo-planar MRI. Magn. Reson. Med. 34, 537-541.

Cordes, D., Haughton, V. M., Arfanakis, K., Wendt, G. J., Turski, P. A., Moritz, C. H., Quigley, M. A., and Meyerand, M. E. (2000). Mapping functionally related regions of brain with functional connectivity MR imaging. AJNR Am. J. Neuroradiol. 21, 1636-1644.

Feinberg, I. (1978). Efference copy and corollary discharge: implications for thinking and its disorders. Schizophr. Bull. 4, 636-640.

Ford, J. M., and Hoffman, R. E. (2012). "Functional brain imaging of auditory hallucinations: from self-monitoring deficits to co-opted neural resources," in The Neuroscience of Hallucinations, eds R. Jardri, A. Cachia, P. Thomas, and D. Pins (New York, NY: Springer).

Ford, J. M., Roach, B. J., Faustman, W. O., and Mathalon, D. H. (2007). Synch before you speak: auditory hallucinations in schizophrenia. Am. J. Psychiatry 164, 458-466.

Fox, M. D., Corbetta, M., Snyder, A. Z., Vincent, J. L., and Raichle, M. E. (2006). Spontaneous neuronal activity distinguishes human dorsal and ventral attention systems. Proc. Natl. Acad. Sci. U.S.A. 103, 10046-10051.

Frith, C. D., and Done, C. J. (1988). Toward a neuropsychology of schizophrenia. Br. J. Psychiatry 153, 437-443.

Gavrilescu, M., Rossell, S., Stuart, G. W., Shea, T. L., Innes-Brown, H., Henshall, K., McKay, C., Sergejew, A. A., Copolov, D., and Egan, G. F. (2010). Reduced connectivity of the auditory cortex in patients with auditory hallucinations: a resting state functional magnetic resonance imaging study. Psychol. Med. 40, 1149-1158.

Green, M. F., Hugdahl, K., and Mitchell, S. (1994). Dichotic listening during auditory hallucinations in patients with schizophrenia. Am. J. Psychiatry 151, 357-362.
Hampson, M., Peterson, B., Skudlarski, P., Gatenby, C., and Gore, J. (2002). Detection of functional connectivity using temporal correlations in MR images. Hum. Brain Mapp. 15, 247-262.

Hampson, M., Tokoglu, F., Sun, Z., Schafer, R. J., Skudlarski, P., Gore, J. C., and Constable, R. T. (2006). Connectivity-behavior analysis reveals that functional connectivity between left BA39 and Broca's area varies with reading ability. Neuroimage 31, 513-519.

Heinks-Maldonado, T. H., Mathalon, D. H., Houde, J. F., Gray, M. Faustman, W. O., and Ford, J. M. (2007). Relationship of imprecise corollary discharge in schizophrenia to auditory hallucinations. Arch. Gen. Psychiatry 64, 286-296.

Hoffman, R. E. (2007). A social deafferentation hypothesis for induction of active schizophrenia. Schizophr. Bull. 33, 1066-1070.

Hoffman, R. E., Anderson, A., Varanko, M., Gore, J., and Hampson, M. (2008). The time course of regional brain activation associated with onset of auditory/verbal hallucinations. Br. J. Psychiatry 193, 424-425.

Hoffman, R. E., Fernandez, T., Pittman, B., and Hampson, M. (2011). Elevated functional connectivity along a corticostriatal loop and the mechanism of auditory/verbal hallucinations in patients with schizophrenia. Biol. Psychiatry 69, 407-414.

Hoffman, R. E., Gueorguieva, R., Hawkins, K. A., Varanko, M., Boutros, N. N., Wu, Y-T., Carroll, K., and Krystal, J. H. (2005). Temporoparietal transcranial magnetic stimulation for auditory hallucinations: safety, efficacy and moderators in a fifty patient sample. Biol. Psychiatry 58, 97-104.

Hoffman, R. E., Hampson, M., Wu, K., Anderson, A., Gore, J., Buchanan, R. J., Constable, T., Hawkins, K., Sahay, N., and Krystal, J. H. (2007). Probing the pathophysiology of auditory hallucinations by combining functional magnetic resonance imaging and transcranial magnetic stimulation. Cereb. Cortex 17, 2733-2743.

Hoffman, R. E., Varanko, M., Gilmore, J., and Mishara, A. L. (2008). Experiential features used by patients with schizophrenia to differentiate voices from ordinary

Addiction Services of the State of Connecticut through its support of the Abraham Ribicoff Research Center at the Connecticut Mental Health Center.

verbal thought. Psychol. Med. 38, $1167-1176$.

Hugdahl, K. (2009). "Hearing voices": auditory hallucinations as failure of top-down control of bottomup perceptual processes. Scand. J. Psychol. 50, 553-560.

Jardri, R., Pouchet, A., Pins, D., and Thomas, P. (2011). Cortical activations during auditory verbal hallucinations in schizophrenia: a coordinate-based meta-analysis. Am. J. Psychiatry 168, 73-81.

Jones, S. R. (2010). Do we need multiple models of auditory verbal hallucinations? Examining the phenomenological fit of cognitive and neurological models. Schizophr. Bull. 36, 566-575.

Kompus, K., Westerhausena, R., and Hugdahl, K. (2011). The "paradoxical" engagement of the primary auditory cortex in patients with auditory verbal hallucinations: a meta-analysis of functional neuroimaging studies. Neuropsychologia 49, 3361-3369.

Lawrie, S. M., Buechel, C., Whalley, H. C., Frith, C. D., Friston, K. J., and Johnstone, E. C. (2002) Reducedfrontotemporal functional connectivity in schizophrenia associated with auditory hallucinations. Biol. Psychiatry 51, 1008-1011.

Lowe, M. J., Mock, B. J., and Sorenson, J. A. (1998). Functional connectivity in single and multislice echoplanar imaging using restingstate fluctuations. Neuroimage 7 , 119-132.

Lui, S., Li, T., Deng, W., Jiang, L., Wu, Q., Tang, H., Yue, Q., Huang, X., Chan, R. C., Collier, D. A., Meda, S. A., Pearlson, G., Mechelli, A., Sweeney, J. A., and Gong, Q. (2010). Short-term effects of antipsychotic treatment on cerebral function in drug-naive first-episode schizophrenia revealed by "resting state" functional magnetic resonance imaging. Arch. Gen. Psychiatry 67, 783-792.

Mechelli, A., Allen, P., Amaro, E. Jr., Fu, C. H., Williams, S. C., Brammer, M. J., Johns, L. C., and McGuire, P. K. (2007). Misattribution of speech and impaired connectivity in patients withauditory verbal hallucinations. Hum. Brain Mapp. 28 , 1213-1222.

Mhuircheartaigh, R. N., RosenornLanng, D., Wise, R., Jbabdi, S. Rogers, R., and Tracey, I. (2010).
Cortical and subcortical connectivity changes during decreasing levels of consciousness in humans: a functional magnetic resonance imaging study using propofol. J. Neurosci. 30 , 9095-9102.

Ojemann, G. A. (1991). Cortical organization of language. J. Neurosci. 11, 2281-2287.

Poulet, E., Brunelin, J., Bediou, B., Bation, R., Forgeard, L., Dalery, J., d'Amato, T., and Saoud, M. (2005). Slow transcranial magnetic stimulation can rapidly reduce resistant auditory hallucinations in schizophrenia. Biol. Psychiatry 57, 188-191.

Price, C. J. (2010). The anatomy of language: a review of $100 \mathrm{fMRI}$ studies published in 2009. Ann. N.Y. Acad. Sci. 1191, 62-88.

Raij, T. T., Valkonen-Korhonen, M., Holi, M., Therman, S., Lehtonen, J., and Hari, R. (2009). Reality of auditory verbal hallucinations. Brain 132, 2994-3001.

Rosen, H. J., Petersen, S. E., Linenweber, M. R., Snyder, A. Z., White, D. A., Chapman, L., Dromerick, A. W., Fiez, J. A., and Corbetta, M. D. (2000). Neural correlates of recovery from aphasia after damage to left inferior frontal cortex. Neurology 55, 1883-1894

Sartorius, N., Shapiro, R., and Jablonsky, A. (1974). The international pilot study of schizophrenia. Schizophr. Bull. 1, 21-35.

Shergill, S. S., Brammer, M. J., Fukuda, R., Williams, S. C. R., Murray, R. M., and McGuire, P. K. (2003). Engagement of brain areas implicated in processing inner speech in people with auditory hallucinations. Br. J. Psychiatry 182, 525-531.

Shmuel, A., and Leopold, D. A. (2008). Neuronal correlates of spontaneous fluctuations in fMRI signals in monkey visual cortex: implications for functional connectivity at rest. Hum. Brain Mapp. 29, 751-761.

Vercammen, A., Knegtering, H., den Boer, J. A., Liemburg, E. J., and Aleman, A. (2010a). Auditory hallucinations in schizophrenia are associated with reduced functional connectivity of the temporoparietal area. Biol. Psychiatry 67, 912-918.

Vercammen, A., Knegtering, $\mathrm{H}$ Liemburg, E. J., den Boer, J. A., and Aleman, A. (2010b). Functional 
connectivity of the temporoparietal region in schizophrenia: effects of rTMS treatment of auditory hallucinations. J. Psychiatr. Res. 44, 725-731.

Xiong, J., Parsons, L. M., Gao, J. H., and Fox, P. T. (1999). Interregional functional connectivity to primary motor cortex revealed using MIR resting state images. Hum. Brain Mapp. 8, 151-156.

Conflict of Interest Statement: The authors declare that the research was conducted in the absence of any commercial or financial relationships that could be construed as a potential conflict of interest.
Received: 29 October 2011; paper pending published: 02 December 2011, accepted: 18 January 2012; published online: 31 January 2012.

Citation: Hoffman RE and Hampson M (2012) Functional connectivity studies of patients with auditory verbal hallucinations. Front. Hum. Neurosci. 6:6. doi: 10.3389/fnhum.2012.00006
Copyright (C) 2012 Hoffman and Hampson. This is an open-access article distributed under the terms of the Creative Commons Attribution Non Commercial License, which permits non-commercial use, distribution, and reproduction in other forums, provided the original authors and source are credited. 